\title{
Non-invasive assessment of ICP during infusion test using Transcranial Doppler Ultrasonography
}

\author{
Danilo Cardim, Brenno Cabella', Joseph Donnelly', Chiara Robba², Marek Czosnyka', Matthew Garnett',

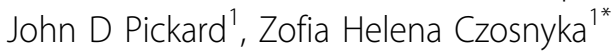 \\ From Hydrocephalus 2015 \\ Banff, Canada. 18-21 September 2015
}

\section{Background}

Transcranial Doppler (TCD) based methods have been used to estimate ICP noninvasively (nICP), however their relative accuracy varies between different types of intracranial hypertension: vasogenic, CSF circulatory or secondary to brain volumetric changes (oedema, contusion, hematoma, etc). This study aimed to compare four nICP methods in a prospective cohort of hydrocephalus patients whose CSF dynamics was investigated using infusion tests involving controllable test-rise of ICP.

\section{Methods}

FV, ICP and non-invasive ABP were recorded in 53 patients diagnosed for hydrocephalus. nICP methods were based on: I) interaction between FV and ABP using blackbox model (nICP_BB); II) diastolic FV (nICP_FVd); III) critical closing pressure (nICP_CrCP) and IV) TCDderived pulsatility index (nICP_PI). Correlation between rise in ICP $(\triangle \mathrm{ICP})$ and $\triangle \mathrm{nICP}$ and averaged correlations for changes in time between ICP and nICP during infusion test were investigated.

\section{Results}

All nICP formulas overestimated ICP at baseline $(\mathrm{p}<0.005)$ : nICP_BB 10.76 (15.08-7.30); nICP_FVd 16.97 (22.56- 11.64); nICP_CrCP 18.34 (20.38-14.89); nICP_PI 16.57 (17.46-16.06). At plateau of ICP during infusion test, only nICP_BB and nICP_PI presented significant difference from ICP. From baseline to plateau, all nICPs estimators increased significantly (paired t-test, $\mathrm{p}<0.05$ ). Correlations between $\triangle \mathrm{ICP}$ and $\triangle \mathrm{nICP}$ were better represented by ICPn_PI and ICPn_BB: 0.45 and $0.30(\mathrm{p}<0.05)$. nICP_FVd and nICP_CrCP presented non-significant correlations: $-0.17(\mathrm{p}=0.21), 0.21(\mathrm{p}=0.13)$. For changes in ICP during individual infusion test ICPn_PI, ICPn_BB and ICPn_FVd presented similar correlations with ICP: $0.39 \pm 0.40, \quad 0.39 \pm 0.43$ and $0.35 \pm 0.41$ respectively. ICPn_CrCP presented a weaker correlation $(\mathrm{R}=0.29 \pm 0.24)$. In those cases where changes of ICP related to vasogenic fluctuations (plateu wabes, B waves) overlapped rise related to CSF infusion, time- correlation between real and estimated ICP seemed to be remarkably better.

\section{Conclusions}

Out of the 4 methods, nICP_PI was the one with best performance for predicting changes in $\triangle \mathrm{ICP}$ during infusion test, followed by nICP_BB. nICP_FVd and nICP_CrCP showed unreliable correlations. Changes of ICP observed during the test were expressed by nICP values with only a moderate correlations. Vasogenic components of ICP seemed to be easier to estimate with TCD, than component related to increased CSF circulation.

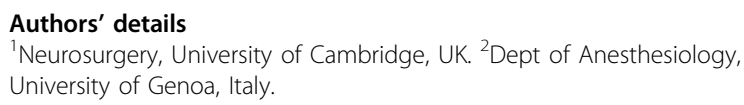

Published: 18 September 2015

doi:10.1186/2045-8118-12-S1-P6

Cite this article as: Cardim et al:: Non-invasive assessment of ICP during infusion test using Transcranial Doppler Ultrasonography. Fluids and Barriers of the CNS 2015 12(Suppl 1):P6.

* Correspondence: zc200@medschl.cam.ac.uk

${ }^{1}$ Neurosurgery, University of Cambridge, UK

Full list of author information is available at the end of the article 